S. Oka

Nagoya Math. J.

Vol. 160 (2000), 181-186

\title{
ON THE UNRAMIFIED COMMON DIVISOR OF DISCRIMINANTS OF INTEGERS IN A NORMAL EXTENSION
}

\author{
SATOMI OKA
}

\begin{abstract}
Let $F$ be an algebraic number field of a finite degree, and $K$ be a normal extension over $F$ of a finite degree $n$. Let $\mathfrak{p}$ be a prime ideal of $F$ which is unramified in $K / F, \mathfrak{P}$ be a prime ideal of $K$ dividing $\mathfrak{p}$ such that $N_{K / F} \mathfrak{P}=\mathfrak{p}^{f}, n=f g$. Denote by $\delta(K / F)$ the greatest common divisor of discriminants of integers of $K$ with respect to $K / F$. Then, $\mathfrak{p}$ divides $\delta(K / F)$ if and only if $\Sigma_{d \mid f} \mu\left(\frac{f}{d}\right) N \mathfrak{p}^{d}<n$.
\end{abstract}

\section{$\S 1$. Introduction}

Let $F$ be an algebraic number field of a finite degree, and $K$ be an extension over $F$ of a finite degree. A basic theorem in the general theory of algebraic number fields says that the greatest common divisor of differents of integers of $K$ with respect to $K / F$ is equal to the different $\mathfrak{d}(K / F)$ of $K / F$. Therefore, the greatest common divisor $\delta(K / F)$ of discriminants of integers of $K$ with respect to $K / F$, as an ideal of $F$, is divisible by the discriminant $d(K / F)=N_{K / F} \mathfrak{d}(K / F)$. It is known, however, that $d(K / F)$ is not always equal to $\delta(K / F)$. In the present paper, we assume that $K / F$ is a normal extension, and will give a necessary and sufficient condition for a prime ideal $\mathfrak{p}$, which is unramified in $K / F$, to divide $\delta(K / F)$. The main theorem is in Section 3.

A prime divisor of $\delta(K / F)$ which does not divide $d(K / F)$ was called "Ausserwesentlicher Diskriminantenteiler" (Dedekind [1]).

\section{$\S 2$. Preliminaries}

1. Throughout the paper, we use standard terminology of number theory as in [2] and [3].

Let $F$ be an algebraic number field of a finite degree, and $K$ be an extension over $F$ of a finite degree $n$. The different $\mathfrak{d}(\alpha, K / F)$ of an element

Received October 26, 1999.

2000 Mathematics Subject Classification: Primary:11R04, Secondary:11R29. 
$\alpha$ of $K$ with respect to $F$ is then defined by $f^{\prime}(\alpha)=\mathfrak{d}(\alpha, K / F)$ where $f(X)$ is the characteristic polynomial of $\alpha=\alpha^{(1)}$ with respect to $K / F$. If $\alpha^{(1)}, \alpha^{(2)}, \cdots, \alpha^{(n)}$ are conjugates of $\alpha$ with respect to $K / F$, the equality $\mathfrak{d}(\alpha, K / F)=\prod_{i \neq 1}\left(\alpha^{(1)}-\alpha^{(i)}\right)$ holds. Furthermore,

$$
\begin{aligned}
d(\alpha, K / F) & =\left|\begin{array}{cccc}
1 & \alpha^{(1)} & \cdots & \alpha^{(1) n-1} \\
1 & \alpha^{(2)} & \cdots & \alpha^{(2) n-1} \\
\cdots & \ldots \ldots \cdots & \\
1 & \alpha^{(n)} & \cdots & \alpha^{(n) n-1}
\end{array}\right|^{2} \\
& =\prod_{i>j}\left(\alpha^{(i)}-\alpha^{(j)}\right)^{2} \\
& =(-1)^{n(n-1) / 2} \prod_{i \neq j}\left(\alpha^{(i)}-\alpha^{(j)}\right) \\
& =(-1)^{n(n-1) / 2} N_{K / F} \mathfrak{d}(\alpha, K / F)
\end{aligned}
$$

implies the relation

$$
d(\alpha, K / F)=(-1)^{n(n-1) / 2} N_{K / F} \mathfrak{d}(\alpha, K / F)
$$

between the different of $\alpha$ and the relative discriminant $d(\alpha, K / F)$ of $\alpha$ with respect to $K / F$.

2. We insert here some elementary facts concerning finite fields.

Let $K_{1}$ be a finite field, and $K_{f}$ be an extension of $K_{1}$ of degree $f$. Then, the Galois group $Z$ of $K_{f} / K_{1}$ is cyclic of order $f$, and, for a divisor $d$ of $f$, there is a unique subfield $K_{d}$ of $K_{f}$ of degree $d$ over $K_{1}$. Denote by $C_{d}$ the set of elements $\gamma$ of $K_{f}$ such that $K_{1}(\gamma)=K_{d}$, and by $c_{d}$ the number of elements of $C_{d}$. Then, $\cup_{d \mid f} C_{d}=K_{f}$ implies $\sum_{d \mid f} c_{d}=q^{f}$, where $q=c_{1}$ is the number of elements of $K_{1}$. Thus, Möbius' inversion formula yields

$$
c_{f}=\sum_{d \mid f} \mu\left(\frac{f}{d}\right) q^{d} .
$$

Every $f$ elements of $C_{f}$ are mutually conjugate under the action of the Galois group $Z$. So, denoting the set of such conjugacy classes of $C_{f}$ by $\tilde{C}_{f}$, the number of elements of $\tilde{C}_{f}$ is $c_{f} / f=M(q, f)$ with

$$
M(q, f)=\frac{1}{f} \sum_{d \mid f} \mu\left(\frac{f}{d}\right) q^{d} .
$$




\section{$\S 3$. Main theorem}

In this article, we assume that $K / F$ is normal with $G=\operatorname{Gal}(K / F)$. Here, as before, $F$ is an algebraic number field of a finite degree, and $K$ is an extension over $F$ of a finite degree $n$. Let now $\mathfrak{o}_{K}$ and $\mathfrak{o}_{F}$ be ring of integers of $K$ and $F$, respectively, $\mathfrak{p}$ a prime ideal of $F$ which is unramified in $K$, and $\mathfrak{P}$ be a prime ideal of $K$ dividing $\mathfrak{p}$. Moreover, let $Z$ be the decomposition group of $\mathfrak{P}, f$ be the order of $Z$, and $\sigma_{1}, \sigma_{2}, \cdots, \sigma_{g}$ be a system of representatives of $Z \backslash G$ fixed once for all with $f g=n$. We then apply (1) to the case where $K_{f}=\mathfrak{o}_{K} / \mathfrak{P}$ and $K_{1}=\mathfrak{o}_{F} / \mathfrak{p}$. We write $C(\mathfrak{P})$ for $C_{f}$ and $\tilde{C}(\mathfrak{P})$ for $\tilde{C}_{f}$ and can see that

$$
M(N \mathfrak{p}, f)=\frac{1}{f} \sum_{d \mid f} \mu\left(\frac{f}{d}\right) N \mathfrak{p}^{d}
$$

is the number of elements of $\tilde{C}(\mathfrak{P})$. Since $\mathfrak{P}$ is an arbitrary divisor of $\mathfrak{p}$ in $K, C\left(\mathfrak{P}^{\sigma}\right)$ and $\tilde{C}\left(\mathfrak{P}^{\sigma}\right)$ for any $\sigma \in G$ are as well-defined as $C(\mathfrak{P})$ and $\tilde{C}(\mathfrak{P})$, and the number of element of $\tilde{C}\left(\mathfrak{P}^{\sigma}\right)$ is equal to that of $C(\mathfrak{P})$ given by $(2)$.

Our main theorem is stated as follows:

THEOREM. Let $F$ be an algebraic number field of a finite degree, and $K$ be a normal extension over $F$ of a finite degree $n$. Let $\mathfrak{p}$ be a prime ideal of $F$ which is unramified in $K / F, \mathfrak{P}$ be a prime ideal of $K$ dividing $\mathfrak{p}$ such that $N_{K / F} \mathfrak{P}=\mathfrak{p}^{f}, n=f g$. Denote by $\delta(K / F)$ the greatest common divisor of discriminants of integers of $K$ with respect to $K / F$, and $M(N \mathfrak{p}, f)$ be as in (2). Then, $\mathfrak{p}$ divides $\delta(K / F)$ if and only if $M(N \mathfrak{p}, f)<g$, or equivalently if and only if $\sum_{d \mid f} \mu\left(\frac{f}{d}\right) N \mathfrak{p}^{d}<n$.

Proof. Meanings of symbols $Z$ and $\sigma_{i}$ being as above, we say that a residue classes represented by $\alpha_{i} \bmod \mathfrak{P}^{\sigma_{i}}$ and by $\alpha_{j} \bmod \mathfrak{P}^{\sigma_{j}},\left(\alpha_{i}, \alpha_{j} \in\right.$ $\left.\mathfrak{o}_{K}\right)$, are conjugate, when there exists an element $\sigma$ of $G=\operatorname{Gal}(K / F)$ such that $\mathfrak{P}^{\sigma_{i} \sigma}=\mathfrak{P}^{\sigma_{j}}$ and $\alpha_{i}^{\sigma} \equiv \alpha_{j} \quad\left(\bmod \mathfrak{P}^{\sigma_{j}}\right)$. In this situation, $\sigma \in \sigma_{i}^{-1} Z \sigma_{j}$ necessarily holds. For each $\sigma_{i}$, the sets $C\left(\mathfrak{P}^{\sigma_{i}}\right)$ and $\tilde{C}\left(\mathfrak{P}^{\sigma_{i}}\right)$ are as welldefined as $C(\mathfrak{P})$ and $\tilde{C}(\mathfrak{P})$ above, and the set of all $C\left(\mathfrak{P}^{\sigma_{i}}\right)$ is divided into $M(N \mathfrak{p}, f)$ conjugacy classes. In particular, the set of conjugacy classes of one $C\left(\mathfrak{P}^{\sigma_{i}}\right)$ coincides with $\tilde{C}\left(\mathfrak{P}^{\sigma_{i}}\right)$, and this set consists of $M(N \mathfrak{p}, f)$ elements either.

Assume now $M \geq g$. Then, there are integers $\alpha_{1}, \alpha_{2}, \cdots, \alpha_{g}$ in $\mathfrak{o}_{K}$ such that the residue class $\alpha_{i} \bmod \mathfrak{P}^{\sigma_{i}}$ belongs to $C\left(\mathfrak{P}^{\sigma_{i}}\right)$ and that $\alpha_{i} \bmod \mathfrak{P}^{\sigma_{i}}$ 
and $\alpha_{j} \bmod \mathfrak{P}^{\sigma_{j}}$ are not conjugate whenever $i \neq j$. Using these integers, we find an integer $\alpha \in \mathfrak{o}_{K}$ satisfying simultaneously

$$
\alpha \equiv \alpha_{i} \quad\left(\bmod \mathfrak{P}^{\sigma_{i}}\right), \quad(i=1,2, \cdots, g) .
$$

Suppose that

$$
\alpha^{\sigma} \equiv \alpha \quad\left(\bmod \mathfrak{P}^{\sigma_{j}}\right)
$$

holds for an element $\sigma \in G,(\sigma \neq 1)$, and for some $j$. Then, taking $\sigma_{i}$ with $\sigma_{i} \sigma=\xi \sigma_{j},(\xi \in Z)$, we have

$$
\alpha_{i}^{\sigma_{i}^{-1} \xi \sigma_{j}} \equiv \alpha_{j} \quad\left(\bmod \mathfrak{P}^{\sigma_{j}}\right)
$$

contrary to the choice of $\alpha_{1}, \alpha_{2}, \cdots, \alpha_{g}$. Thus, $\alpha-\alpha^{\sigma}$ is not divisible by any $\mathfrak{P}^{\sigma_{j}}$, and therefore is prime to $\mathfrak{p}$. From this follows that $\mathfrak{p}$ does not divide $\delta(K / F)$.

Assume conversely $M<g$. Then (3) should hold for $\sigma=\sigma_{i}^{-1} \xi \sigma_{j}$ with some $\sigma_{i}, \sigma_{j},(i \neq j)$ and $\xi \in Z$, whenever $\alpha$ is an integer in $\mathfrak{o}_{K}$ such that $\alpha \bmod \mathfrak{P}_{i}$ belongs to $C\left(\mathfrak{P}^{\sigma_{i}}\right)$ for every $i$. This means that the discriminant of such an $\alpha$ with respect to $K / F$ is divisible by $\mathfrak{p}$. If $\alpha$ is an integer in $\mathfrak{o}_{K}$, and $\alpha \bmod \mathfrak{P}^{\sigma_{i}}$ does not belong to $C\left(\mathfrak{P}^{\sigma_{i}}\right)$ for some $i$, then

$$
\alpha^{\sigma_{i}^{-1} \xi \sigma_{i}} \equiv \alpha \quad\left(\bmod \mathfrak{P}^{\sigma_{i}}\right)
$$

holds with an element $\xi$ of $Z,(\xi \neq 1)$, which implies (3) with $\sigma=\sigma_{i}^{-1} \xi \sigma_{i} \neq$ 1. From all these arguments, we can conclude that the discriminant of an integer $\alpha$ in $\mathfrak{o}_{K}$ is divisible by $\mathfrak{p}$ regardless of its residue class $\bmod \mathfrak{p}$. Hence, $\mathfrak{p}$ divides $\delta(K / F)$.

Corollary 1. Assume that the prime ideal in the Theorem decomposes completely in $K$. Then, $\mathfrak{p}$ divides $\delta(K / F)$ if and only if $N \mathfrak{p}<n$.

Proof. In this case, $f=1$, and $\sum_{d \mid f} \mu\left(\frac{f}{d}\right) N \mathfrak{p}^{d}=N \mathfrak{p}$.

Corollary 2. If the prime ideal $\mathfrak{p}$ in the Theorem satisfies $N \mathfrak{p} \geq n$, then $\mathfrak{p}$ dose not divide $\delta(K / F)$.

Proof. Put $N \mathfrak{p}=q$. Then,

$$
\begin{aligned}
\sum_{d \mid f} \mu\left(\frac{f}{d}\right) q^{d} & \geq q^{f}-\sum_{d \mid f, d<f} q^{d} \geq q^{f}-\left(q^{f-1}+q^{f-2}+\cdots+q\right) \\
& =q-q \frac{q^{f-1}-1}{q-1} \geq q^{f}-q\left(q^{f-1}-1\right)=q \geq n
\end{aligned}
$$




\section{$\S 4$. Examples}

1. Let $K$ be a composite of a finite number $(>1)$ of quadratic fields over $\mathbf{Q}=F$ in which 2 is unramified. Then, the degree $f$ of a prime factor of 2 in $K$ is either 1 or 2 , and $n=(K: \mathbf{Q}) \geq 4$. If $f=1$, then Corollary 1 shows that 2 divides $\delta(K / \mathbf{Q})$. If $f=2$, then the number $M(N \mathfrak{p}, f)$ in the Theorem is $\frac{1}{2}\left(2^{2}-2\right)=1$. Since $g=\frac{n}{2} \geq 2$, the Theorem implies that 2 divides $\delta(K / \mathbf{Q})$. Namely, 2 always divides $\delta(K / \mathbf{Q})$, whenever $K$ is a composite of quadratic fields in which 2 is unramified.

2. Let $p$ be a prime number, and $l$ be a prime number dividing $p^{3}-1$. Then, $p$ decomposes completely in the subfield $K$ of the cyclotomic field $\mathbf{Q}\left(e^{(2 \pi i) / l}\right)$ with the property $(K: \mathbf{Q})=\frac{1}{3}(l-1)$. If here moreover $\frac{1}{3}(l-1)>$ $p$, then it follows from Corollary 1 that $p$ divides $\delta(K / \mathbf{Q})$.

A few actual numerical examples are:

\begin{tabular}{c|ccccc}
$p$ & 3 & 5 & 7 & 11 & 13 \\
\hline$l$ & 13 & 31 & - & - & 61
\end{tabular}

3. Let $K / \mathbf{Q}$ be normal of degree 4 . If $K / \mathbf{Q}$ is not cyclic and 2 is unramified, then example 1 shows that 2 divides $\delta(K / \mathbf{Q})$. Even if $K / \mathbf{Q}$ is cyclic, $\sum_{d \mid f} \mu\left(\frac{f}{d}\right) 2^{d}$ is 2 for $f=1$ and 2 . Therefore, 2 divides $\delta(K / \mathbf{Q})$, unless 2 remains prime in $K$. If 3 is completely decomposed in $K$, then Corollary 1 implies that 3 divides $\delta(K / \mathbf{Q})$. But, if 3 is not completely decomposed and unramified, then $\sum_{d \mid f} \mu\left(\frac{f}{d}\right) 3^{d}=3^{4}-3^{2}$ or $3^{2}-3$, and is bigger than 4 . So, by the Theorem, 3 does not divide $\delta(K / \mathbf{Q})$. The unramified primes bigger than 3 do not divide $\delta(K / \mathbf{Q})$ as a consequence of Corollary 2 .

Acknowledgements. The author expresses her thanks to Professor Tomio Kubota for his advice and encouragement.

\section{REFERENCES}

[1] R. Dedekind, Über den Zusammenhang zwuschen der Theorie der Ideale und der Theorie der höheren Kongruenzen, Abh.der König. Gesell. der Wiss. zu Göttingen, 23 (1878), 1-23, Complete works, Chelsea, 1969.

[2] S. Lang, Algebraic number theory, Addison-Wesley, 1970.

[3] E. Weiss, Algebraic number theory, AcGraw-Hill, 1963. 
Department of Mathematics Meijo University

Shiogamaguchi 1-501, Tenpakuku

Nagoya, 468-8502, Japan

d3991001@meijo-u.ac.jp 